\title{
DOUBLE-BUNDLE ANATOMICAL RECONSTRUCTION OF THE ANTERIOR CRUCIATE LIGAMENT: A PROSPECTIVE STUDY WITH TWO-YEAR FOLLOW-UP
}

Julio Cesar Gali', Maurício Sante Bettio Mod², Hélio Massahiro Mimura², Walberto Kushiyama ${ }^{3}$

\begin{abstract}
Objective: To prospectively evaluate the results from double-bundle reconstruction of the anterior cruciate ligament, among patients at our clinic, by means of the 2000 protocol of the International Knee Documentation Committee (IKDC). Study Design: Case series; level of evidence IV. Methods: Fifty-eight patients who underwent anatomical reconstruction of the anterior cruciate ligament using an autologous flexor tendon graft by means of the double-bundle technique were evaluated in accordance with the IKDC 2000 protocol. The
\end{abstract}

patients' ages ranged from 17 to 58 years, with a mean of 35.2 years. The follow-up ranged from 24 to 37 months (mean of 28.9 months). Results: Postoperatively, $89.65 \%$ of the pivot-shift test findings were negative. In the final evaluation, $44(75.86 \%)$ of the patients' knees were graded as normal, $13(22.41 \%)$ as nearly normal and one $(1.72 \%)$ as abnormal. Conclusion: The technique used was effective in promoting restoration of joint stability, without compromising mobility.

Keywords - Anterior Cruciate Ligament; Orthopedic Procedures; Treatment Results

\section{INTRODUCTION}

There is great interest in surgical reconstruction of the anterior cruciate ligament (ACL), given that the long-term results from conservative treatment are unsatisfactory. Only a low percentage of patients return to the same level as before the injury, without limitations, and there are frequent complaints of instability, leading to the need for secondary reconstruction ${ }^{(1)}$. There is also a likelihood of future osteoarthritis in 60 to $100 \%$ of the cases, 20 years later ${ }^{(2)}$.

The surgical technique most commonly used to treat this condition is arthroscopic reconstruction with a single bundle, such that the femoral tunnel is made through the tibial tunnel. This favors construction of the femoral tunnel at a higher location on the intercondile $^{(3)}$, i.e. differing from the anatomical description of the femoral insertion site ${ }^{(4)}$.

Evaluations on the percentage success of ACL reconstructions using a single bundle using meta-analysis have shown rates of $69 \%$ to $95 \%{ }^{(5,6)}$. An evidence-based study showed that radiographic signs of osteoarthritis occur in $50 \%$ of the patients undergoing ACL surgical reconstruction, regardless of the technique used ${ }^{(7)}$.

\footnotetext{
$1-\mathrm{PhD}$ in Orthopedics and Traumatology from the USP School of Medicine. Attending Physician at Apex Orthopedics and Traumatology and at the Unimed Hospital, Sorocaba. 2 - Attending Physician at Apex Orthopedics and Traumatology and at the Unimed Hospital, Sorocaba.

3 - MSc in Orthopedics and Traumatology from Unifesp.

Correspondence: Av. Eugênio Salerno 387 - 18035-430 - Sorocaba, SP. Email: juliogali@apice.med.br
}

Work received for publication: March 2, 2010; accepted for publication: October 29, 2010. 
These findings have motivated research on new techniques that aim towards reconstructing the ACL in an anatomical manner, using a double bundle. Basic science studies have demonstrated advantages from double-bundle reconstruction, in comparison with single-bundle reconstruction ${ }^{(8-10)}$, as have prospective and randomized studies ${ }^{(11-17)}$.

The aim of our study was to analyze the results from anatomical reconstruction of the ACL using a double bundle, in our setting, using the protocol of the International Knee Documentation Committee $2000^{(18)}$.

\section{MATERIAL AND METHODS}

We prospectively followed up 58 patients who underwent anatomical reconstructions of the ACL of the knee, using autologous grafts from the flexor tendons, by means of the double-bundle technique, starting in March 2006. All the patients originated from our private clinic.

The patients were assessed at the following times after the operation: one week; 30 days; two, three, four and six months; and one and two years. At these return visits, we routinely assessed and recorded data on the patients' stability and degree of mobility.

For this study, patients were excluded if they presented the following criteria: bilateral surgery, previous ligament surgery or associated ligament injuries.

The length of the follow-up ranged from 24 to 37 months, with a mean of 28.9 months. The patients' ages ranged from 17 to 58 years, with a mean of 35.2 years. There were four female patients $(6.89 \%)$ and 54 male patients $(93.10 \%)$. The right side was affected in 32 cases $(55.17 \%)$ and the left side in 26 cases $(44.82 \%)$.

\section{Surgical technique}

We made an incision of around $4 \mathrm{~cm}$ in the proximal and medial thirds of the lower leg. With the aid of an extractor, we removed the flexor tendons of the gracilis and semitendinosus muscles. The semitendinosus tendon was used for the anteromedial bundle of the ACL graft, while the gracilis tendon was used for the posterolateral bundle.

For simplification, we routinely used an Endobutton 25 for the anteromedial bundle and an Endobutton 20 for the posterolateral bundle. We performed arthroscopy for diagnosis and for treating the meniscal or chondral lesions.

The femoral and tibial insertions of the antero- medial and posterolateral bundles were marked with radiofrequency and kept intact in order to preserve the vascularization and proprioception.

Using the same instrument, we demarcated the tibial insertions. The tibial insertion of the posterolateral bundle was located anteriorly and medially to the posterior root of the lateral meniscus, and anteriorly and laterally to the posterior cruciate ligament (PCL). The insertion site for the anteromedial tibial bundle was slightly anteriorly and medially to the conventional location for the tibial tunnel used in the single-bundle technique ${ }^{(19)}$.

An anteromedial accessory portal was established using an Abocath 14, under direct viewing, inferiorly and medially to the standard anteromedial portal. Its positioning was critical for obtaining the correct path for the drill bit, to construct the posterolateral femoral tunnel, in order to avoid injuring the surface of the medial femoral condyle or the medial meniscus, while drilling.

The posterolateral femoral tunnel was demarcated $5 \mathrm{~mm}$ posteriorly to the anterior cartilage of the lateral femoral condyle, and $3 \mathrm{~mm}$ superiorly to the inferior cartilage of the latter, with the knee flexed at $90^{\circ}$. After determining the ideal point, we drilled a hole using a bone pick.

The knee was flexed at $110^{\circ}$ to protect the common fibular nerve. The posterolateral femoral tunnel was drilled through the anteromedial accessory portal, using a $5 \mathrm{~mm}$ bit, by means of crossing the lateral cortical bone of the lateral femoral condyle.

To construct the tibial tunnels, the drilling guide was adjusted to the setting of $55^{\circ}$. We kept a bone bridge of at least $1 \mathrm{~cm}$ between the guidewires (Figure 1).

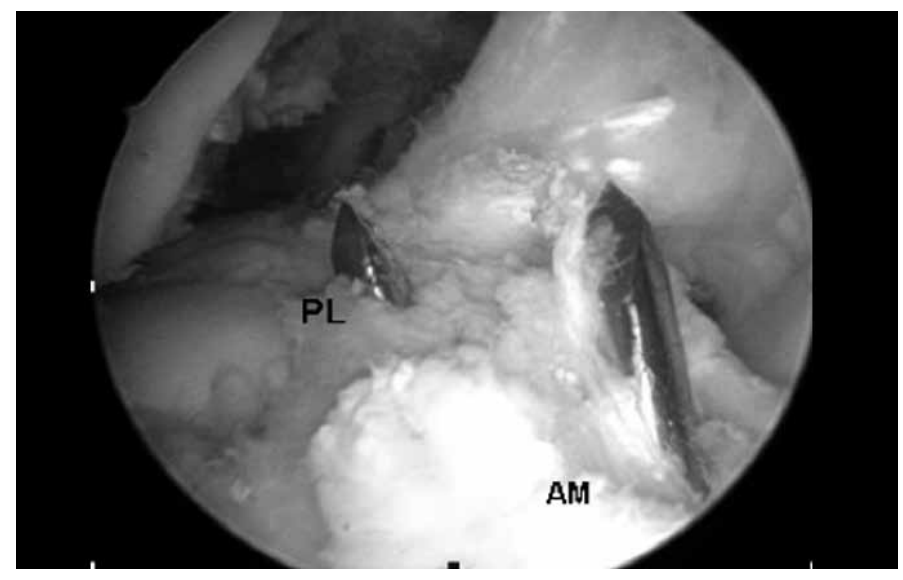

Figure 1 - Intraoperative view of the guidewires on the tibial joint surface. 
The anteromedial femoral tunnel was constructed at depth in relation to the edge of the posterolateral tunnel. The guidewire could be introduced transtibially, through the posterolateral tunnel, or through the anteromedial accessory portal of the arthroscopy. Our preference was for a transtibial route, which left the femoral tunnels divergent.

The anteromedial femoral tunnel was drilled using a $5 \mathrm{~mm}$ drill bit and would cross over the femoral cortical bone. Subsequently, we used a $7 \mathrm{~mm}$ bit to drill into the lateral femoral condyle to a depth of 35 mm (Figure 2).

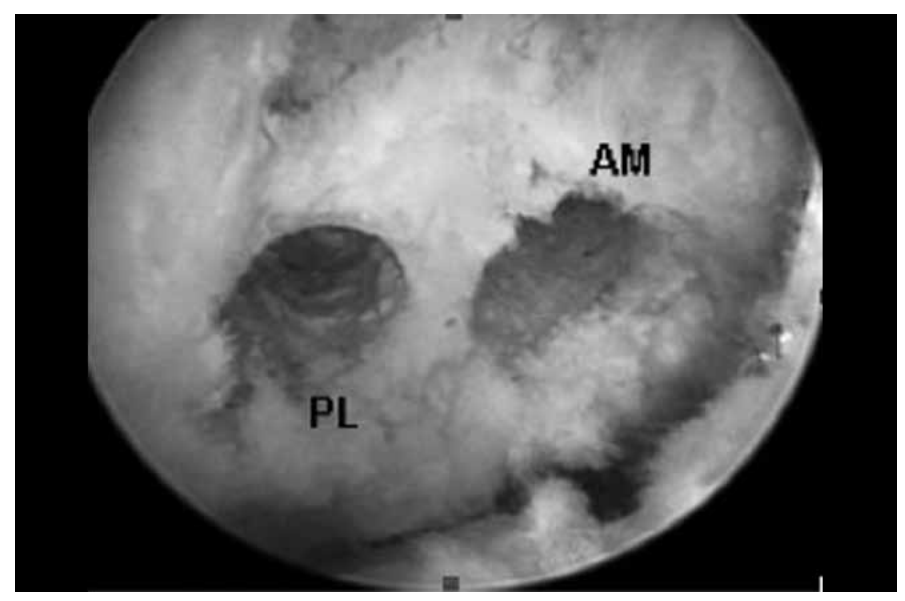

Figure 2 - View of the anteromedial (AM) and posterolateral (PL) femoral tunnels, through the anteromedial arthroscopic portal.

A drilled guidewire with a Vicryl 1 wire at two orifices was passed through the anteromedial accessory portal, through the posterolateral femoral tunnel and through the lateral skin of the thigh, while a double end of the Vicryl 1 was kept inside the joint. Next, this end was pulled into the posterolateral tibial tunnel with the aid of a hook, and brought out to the external region of the lower leg. Two wires of different colors were used, to make it easier to identify each component.

Following this, the posterolateral graft was passed through the tibial and femoral tunnels, and the Endobutton was overturned, thus providing femoral fixation. The anteromedial graft was then passed through the corresponding tunnels. The Endobutton was overturned, similarly to the posterolateral graft.

Before fixation, each component of the graft was pretensioned through flexion-extension movements of the knee, 25 times, using manual tension.

We used $25 \times 8$ metal interference screws to fix the graft in the anteromedial tunnel, and $30 \times 8$ screws to fix the graft in the posterolateral tunnel. Absorbable interference screws of similar lengths and diameters could also be used.

The anteromedial graft was fixed with the knee flexed at $45^{\circ}$. The posterolateral graft was fixed with the knee flexed at $15^{\circ}$. In both situations, we undertook manual tensioning of the wires at the tibial end of the graft (Figures 3 and 4).

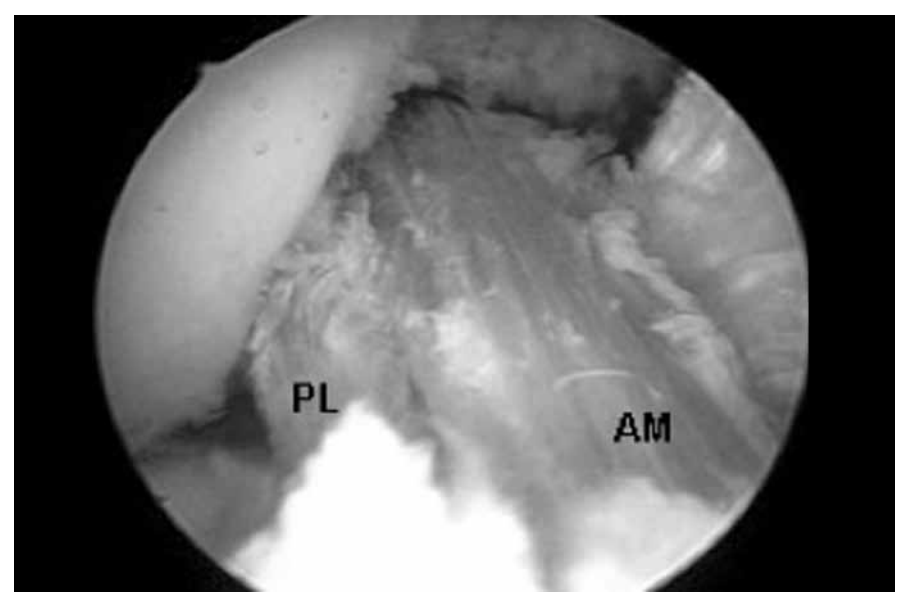

Figure 3 - Arthroscopic view of the anteromedial (AM) and posterolateral $(\mathrm{PL})$ bundles, through the anterolateral portal.

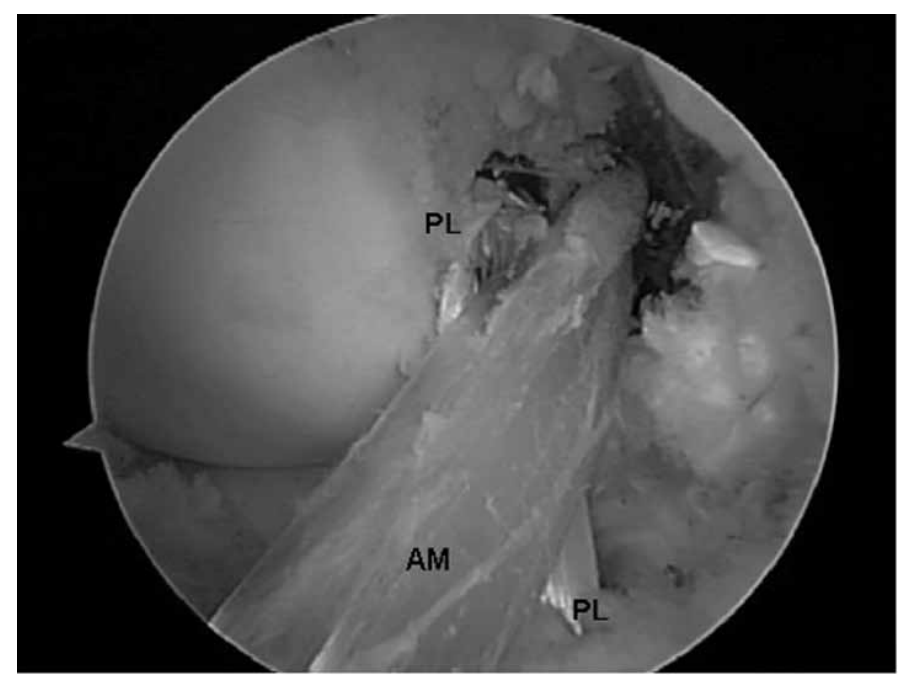

Figure 4 - Arthroscopic view of the anteromedial (AM and posterolateral (PL) bundles, through the anteromedial portal.

Source: Ápice Ortopedia e Traumatologia

\section{Rehabilitation}

We allowed partial body weight-bearing, with the aid of crutches, on the day after the operation, the progression to full weight-bearing was done according to the pain levels. The use of crutches was withdrawn around the seventh day, at which time $90^{\circ}$ of flexion 
can usually be achieved. Gentle stretching of the hamstrings should be started immediately, to minimize painful adherences.

In a general manner, we followed the same parameters as described previously ${ }^{(20)}$.

\section{Assessment of the results}

The postoperative results were assessed using the protocol of the International Knee Documentation Committee 2000.

\section{RESULTS}

For 25 patients, we performed partial medial meniscectomy in the same surgical procedure as the ligament reconstruction. In three, we performed partial lateral meniscectomy, and in another nine cases, partial resection of both menisci was done. In no case was any reconstructive procedure performed on the joint cartilage.

In group 1 (joint effusion), 54 of the patients' knees (93.1\%) were graded as normal (A) and four (6.89\%) as close to normal (B); in group 2 (passive motor deficit), 53 (91.37\%) were classified as normal (A) and five $(8.62 \%)$ as close to normal (B); in group 3 (ligament examination), 52 knees $(89.65 \%)$ were classified as normal (A), five (8.62\%) as close to normal (B) and one (1.72\%) as abnormal (C).

In the final assessment, $44(75.86 \%)$ of the patients' knees were graded as normal (A), 13 (22.41\%) as close to normal (B) and one (1.72\%) as abnormal (C) (Figure 5).

There were no significant complications and we did not register any cases of infection.

\section{DISCUSSION}

The ACL is composed of two anatomical bundles: the anteromedial and posterolateral bundles, according to their tibial insertion ${ }^{(4)}$. Functionally, the anteromedial bundle becomes tense under flexion, which in this position the posterolateral bundle relaxes; conversely, the posterolateral bundle becomes tensioned under extension, while the anteromedial bundle relaxes $^{(21)}$.

Recent studies using navigation for primary reconstruction of the ACL using a double bundle have found that the anteromedial and posterolateral components control both the anterior translation and the rotation during the pivot shift test ${ }^{(22,23)}$.

Rev Bras Ortop. 2011;46(1):31-6

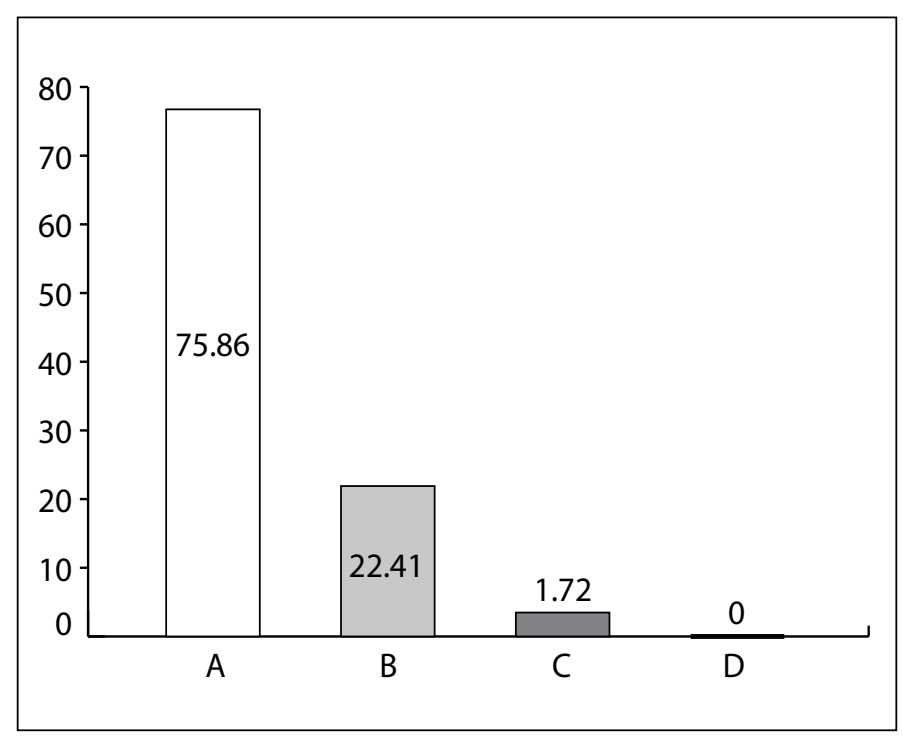

Figure 5 - Results from the final assessment using IKDC 2000.

In the technique described above, the anteromedial portal provided better viewing of the lateral femoral condyle and the femoral insertions of the ACL than did the AL portal, which has traditionally been used for these reconstructions. The arthroscope was inserted in the anteromedial portal, while the anteromedial accessory portal was used to make the marks for the ACL insertions in the lateral femoral condyle, and for drilling the posterolateral femoral tunnel.

We preferentially made the anteromedial femoral tunnel through the posterolateral tibial tunnel, because this gave the possibility of producing longer and divergent tunnels. In situations in which this has not been possible, we have also made the anteromedial femoral tunnel through the anteromedial accessory portal, which generally results in a shorter tunnel that is parallel to the posterolateral femoral tunnel.

$\mathrm{Fu}$ et $\mathrm{al}^{(24)}$ reported that accuracy in constructing the anteromedial femoral tunnel through the anteromedial tibial tunnel was only achieved in $10 \%$ of the cases. On the other hand, accuracy was achieved through the posterolateral tibial tunnel in $60 \%$ of the cases, and through the anteromedial accessory portal in $100 \%$ of the times for which this was the access chosen.

So far, in all our cases, the posterolateral femoral tunnel could, in a simplified manner, be made just by using a $5 \mathrm{~mm}$ drill bit, without any need to increase the diameter of the portion that was more proximal to the joint. The graft made from the gracilis had a sufficiently narrow diameter to allow it to pass through the tunnel and allow the Endobutton to be overturned. 
It is important to highlight that there is no need for special guides, since this is an insertion site technique, thereby ensuring that the reconstruction is not affected by patients' anatomical variations or by other intraarticular references.

In our assessment using IKDC, four patients $(6.89 \%)$ reported that they had some swelling. Full joint movement was obtained in the cases of 53 $(91.37 \%)$ of the knees. Without statistical proof, we observed that with the double-bundle technique, the patients had a tendency to achieve full knee flexion earlier.

In the ligament examinations, the pivot shift test was negative in 44 of the patients' knees (89.65\%); in five cases $(8.62 \%)$, the result from the pivot shift test was $1+$. The patient graded as $\mathrm{C}$ (pivot shift $2+$ ) suffered a traumatic failure after slipping on a smooth floor, two months after the operation.

In the final assessment, $44(75.86 \%)$ of our patients' knees were graded as A, $13(22.41 \%)$ as B and one $(1.72 \%)$ as $\mathrm{C}$.

In a prospective study without a control group, like in our study, $\mathrm{Fu}$ et $\mathrm{al}^{(24)}$ assessed 73 patients with a minimum follow-up of two years. They found that $65 \%$ had a normal result from the Lachman test and $33 \%$ were close to normal. In the pivot shift test, $94 \%$ were considered to be normal and $6 \%$ were close to normal.

In 2008, Järvelä et $\mathrm{al}^{(14)}$ reported that double-bundle reconstruction produced better rotational stability. They suggested that this might protect the knee from new lesions that could produce graft failure. In the final assessment using IKDC, $59 \%$ of the patients were classified as normal, $36 \%$ as close to normal and $4 \%$ as abnormal.

Siebold et al ${ }^{(15)}$ also published their results in 2008. In the assessment using IKDC, the group treated with a double bundle presented grade $\mathrm{A}$ in $78 \%$ of the cases, $\mathrm{B}$ in $19 \%$ and $\mathrm{D}$ in $3 \%$. This last case was a traumatic recurrence of rupture.

In 2010, Aglietti et al ${ }^{(17)}$ assessed 35 patients with chronic ACL injuries who were treated with doublebundle reconstruction. In the final assessment, these authors found that $80 \%$ of the patients were classified as normal, $17 \%$ as close to normal and $3 \%$ as abnormal.

Song et $\mathrm{l}^{(25)}$ used a navigation system to measure the intraoperative rotational stability and concluded that reconstruction using a double bundle effectively reduced the residual pivot shift after ACL reconstruction.

Even if the duration of the operation does not increase significantly ${ }^{(25)}$, ACL reconstruction surgery using a double bundle is not for surgeons who only occasionally operate on knees. Regarding this topic, Lyman et al ${ }^{(26)}$ concluded that the risk involved in reoperating the ACL is higher in cases treated by surgeons whose volume of surgery is low.

It is important to emphasize that situations that are more appropriate for ACL reconstruction using a single bundle exist, such as: ACL insertion less than $14 \mathrm{~mm}$, narrow intercondyle (less than $12 \mathrm{~mm}$ ), open growth plate, grade III arthrosis or higher, multiple ligament lesions and severe bone contusion ${ }^{(19)}$.

Perhaps the most important "side effect" from describing the technique and results from double-bundle reconstruction is the raising of awareness of the need to respect the anatomical ACL insertion sites. In other words, even when a single bundle is used, the femoral insertion should be oblique, done through the anteromedial accessory portal. Femoral tunnel construction using a transtibial route should be avoided. Reconstruction respecting each patient's anatomy becomes necessary to enable knee function with the reconstructed ACL to be closer to normal.

In this regard, Scanlan et $\mathrm{al}^{(27)}$ concluded that graft placement plays a critical role in restoring the normal gait mechanism after ACL reconstruction, and that this may partially explain the incidence of premature arthrosis over the long term.

Despite the biomechanical advantages and the encouraging initial results, there is a need for equipment that can quantify rotational slackness and for long-term multicenter prospective studies comparing truly anatomical homogenous techniques for singlebundle or double-bundle reconstruction, with precise descriptions, in order to be able prove superior functional results, diminish the likelihood of new meniscal lesions and joint cartilage lesions, and furthermore, to diminish the risk of future osteoarthrosis caused by ACL failure.

\section{CONCLUSION}

Double-bundle reconstruction was, in our hands, a safe and effective technique for restoring joint stability after ACL injury, without prejudicing the degree of mobility and with low incidence of complications. 


\section{REFERENCES}

1. Scavenius M, Bak K, Hansen S, Norring K, Jensen KH, Jorgensen U. Isolated total ruptures of the anterior cruciate ligament--a clinical study with long-term follow-up of 7 years. Scand J Med Sci Sports. 1999;9(2):114-9.

2. Louboutin H, Debarge R, Richou J, Selmi TA, Donell ST, Neyret P, et al. Osteoarthritis in patients with anterior cruciate ligament rupture: a review of risk factors. Knee. 2009;16(4):239-44.

3. Arnold MP, Kooloos J, van Kampen A. Single-incision technique misses the anatomical femoral anterior cruciate ligament insertion: a cadaver study. Knee Surg Sports Traumatol Arthrosc. 2001;9(4):194-9.

4. Girgis FG, Marshall JL, Al Monajem, AR. The cruciate ligaments of the knee joint. Anatomical, functional and experimental analysis. Clin Orthop Relat Res. 1975;(106):216-31.

5. Freedman KB, D'Amato MJ, Nedeff DD, Kaz A, Bach BR. Arthroscopic anterior cruciate ligament reconstruction: a metaanalysis comparing patellar tendon and hamstring tendon autografts. Am J Sports Med. 2003;31(1):2-11.

6. Yunes M, Richmond JC, Engels EA, Pinczewski, LA. Patellar versus hamstring tendons in anterior cruciate ligament reconstruction: A meta-analysis. Arthroscopy. 2001;17(3):248-57.

7. Andersson D, Samuelsson K, Karlsson J. Treatment of anterior cruciate ligament injuries with special reference to surgical technique and rehabilitation: an assessment of randomized controlled trials. Arthroscopy. 2009;25(6):653-85.

8. Yagi M, Wong EK, Kanamori A, Debski RE, Fu FH, Woo SL. Biomechanica analysis of an anatomic anterior cruciate ligament reconstruction. Am J Sports Med. 2002;30(5):660-6.

9. Petersen W, Tretow H, Weimann A, Herbort M, Fu FH, Raschke M, et al. Biomechanical evaluation of two techniques for double-bundle anterior cruciate ligament reconstruction: one tibial tunnel versus two tibial tunnels. Am J Sports Med. 2007;35(2):228-34.

10. Morimoto Y, Ferretti M, Ekdahl M, Smolinski P, Fu FH. Tibiofemoral joint contact area and pressure after single- and double-bundle anterior cruciate ligament reconstruction. Arthroscopy. 2009;25(1):62-9.

11. Yasuda $\mathrm{K}$, Kondo $\mathrm{E}$, Ichiyama $\mathrm{H}$, Tanabe $\mathrm{Y}$, Tohyama $\mathrm{H}$. Clinical evaluation of anatomic double-bundle anterior cruciate ligament reconstruction procedure using hamstring tendon grafts: comparisons among 3 different procedures. Arthroscopy. 2006;22(3):240-51.

12. Yagi M, Kuroda R, Nagamune K, Yoshiya S, Kurosaka M. Double-bundle ACL reconstruction can improve rotational stability. Clin Orthop Relat Res. 2007;(454): 100-7.

13. Muneta T, Koga H, Mochizuki T, Ju YJ, Hara K, Nimura A, et al. A prospective randomized study of 4-strand semitendinosus tendon anterior cruciate ligament reconstruction comparing single-bundle and double-bundlentechniques. Arthroscopy. 2007;23(6):618-28.

14. Järvelä T, Moisala AS, Sihvonen R, Järvelä S, Kannus P, Järvinen M. Double-bundle anterior cruciate ligament reconstruction using hamstring autografts and bioabsorbable interference screw fixation: prospective, randomized, clinical study with 2-year results. Am J Sports Med. 2008;36(2):290-7.

15. Siebold R, Dehler C, Ellert T. Prospective randomized comparison of double-bundle versus single-bundle anterior cruciate ligament reconstruction. Arthroscopy. 2008;24(2):137-45.

16. Ibrahim SAR, Hamido F, Al Misfer AK, Mahgoob A, Ghafar SA, Alhran H. Anterior cruciate ligament reconstruction using autologous hamstring double bundle graft compared with single bundle procedures. J Bone Joint Surg Br. 2009;91(10):1310-5.

17. Aglietti P, Giron F, Losco M, Cuomo P, Ciardullo A, Mondanelli N. Comparison between single-and double-bundle anterior cruciate ligament reconstruction: a prospective, randomized, single-blinded clinical trial. Am J Sports Med. 2010;38(1):25-34.

18. Irrgang JJ, Anderson AF, Boland AL, Harner CD, Kurosaka M, Neyret $P$, et al. Development and validation of the international knee documentation committee subjective knee form. Am J Sports Med. 2001;29(5):600-13.

19. Van Eck CF, Lesniak BP, Schreiber VM, Fu, FH. Anatomic Single- and Double-Bundle Anterior Cruciate Ligament Reconstruction Flowchart. Arthroscopy 2010;26(2):258-68.

20. Gali JC, Camanho GL. A reabilitação acelerada após reconstrução do ligamento cruzado anterior com enxerto de tendão patelar é segura? Rev Bras Ortop. 1998;33(8):645-50.

21. Amis AA, Dawkins GP. Functional anatomy of the anterior cruciate ligament. Fibre bundle actions related to ligament replacements and injuries. J Bone Joint Surg Br. 1991;73(2):260-7.

22. Ishibashi $Y$, Tsuda E, Yamamoto $Y$, Tsukada H, Toh S. Navigation evaluation of the pivot-shift phenomenon during double-bundle anterior cruciate ligament reconstruction: is the posterolateral bundle more important? Arthroscopy. 2009;25(5):488-95.

23. Robinson J, Carrat L, Granchi C, Colombet P. Influence of anterior cruciate ligament bundles on knee kinematics: clinical assessment using computer-assisted navigation. Am J Sports Med. 2007;35(12):2006-13.

24. Fu FH, Shen W, Starman JS, Okeke N, Irrgang JJ. Primary anatomic double-bundle anterior cruciate ligament reconstruction: a preliminary 2-year prospective study. Am J Sports Med. 2008;36(7):1263-74.

25. Song EK, Oh LS, Gill TJ, Li G, Gadikota HR, Seon JK. Prospective comparative study of anterior cruciate ligament reconstruction using the double-bundle and single-bundle techniques. Am J Sports Med. 2009;37(9)1705-11.

26. Lyman S, Koulouvaris P, Sherman S, Do H, Mandl LA, Marx RG. Epidemiology of anterior cruciate ligament reconstruction. trends, readmissions, and subsequent knee surgery. J Bone Joint Surg Am. 2009;91(10):2321-8.

27. Scanlan SF, Blazek K, Chaudhari AMW, Safran MR, Andriacchi TP. Graft orientation influences the knee flexion moment during walking in patients with anterior cruciate ligament reconstruction. Am J Sports Med. 2009;37(11):2173-8. 